\title{
A randomised controlled trial of acceptance and commitment therapy (ACT) for psychosis: study protocol
}

Neil Thomas ${ }^{1,2}$, Frances Shawyer ${ }^{3,4}$, David J Castle ${ }^{5,6}$, David Copolov ${ }^{7,8,9}$, Steven C Hayes ${ }^{10}$ and John Farhall ${ }^{4,11^{*}}$

\begin{abstract}
Background: Cognitive behavior therapy for psychosis has been a prominent intervention in the psychological treatment of psychosis. It is, however, a challenging therapy to deliver and, in the context of increasingly rigorous trials, recent reviews have tempered initial enthusiasm about its effectiveness in improving clinical outcomes. Acceptance and commitment therapy shows promise as a briefer, more easily implemented therapy but has not yet been rigorously evaluated in the context of psychosis. The purpose of this trial is to evaluate whether Acceptance and Commitment Therapy could reduce the distress and disability associated with psychotic symptoms in a sample of community-residing patients with chronic medication-resistant symptoms.

Methods/Design: This is a single (rater)-blind multi-centre randomised controlled trial comparing Acceptance and Commitment Therapy with an active comparison condition, Befriending. Eligible participants have current residual hallucinations or delusions with associated distress or disability which have been present continuously over the past six months despite therapeutic doses of antipsychotic medication. Following baseline assessment, participants are randomly allocated to treatment condition with blinded, post-treatment assessments conducted at the end of treatment and at 6 months follow-up. The primary outcome is overall mental state as measured using the Positive and Negative Syndrome Scale. Secondary outcomes include preoccupation, conviction, distress and disruption to life associated with symptoms as measured by the Psychotic Symptom Rating Scales, as well as social functioning and service utilisation. The main analyses will be by intention-to-treat using mixed-model repeated measures with non-parametric methods employed if required. The model of change underpinning ACT will be tested using mediation analyses.
\end{abstract}

Discussion: This protocol describes the first randomised controlled trial of Acceptance and commitment therapy in chronic medication-resistant psychosis with an active comparison condition. The rigor of the design will provide an important test of its action and efficacy in this population.

Trial registration: Australian New Zealand Clinical Trials Registry: ACTRN12608000210370. Date registered: 18 April 2008

Keywords: Randomised controlled trial (RCT), Psychosis, Schizophrenia, Auditory hallucinations, Delusions, Positive symptoms, Negative symptoms, Acceptance and commitment therapy (ACT), Psychological therapy, Befriending

\footnotetext{
* Correspondence: j.farhall@latrobe.edu.au

${ }^{4}$ School of Psychological Science, La Trobe University, Melbourne, Victoria

3086, Australia

${ }^{11}$ NorthWestern Mental Health, Royal Melbourne Hospital, Melbourne,

Victoria 3050, Australia

Full list of author information is available at the end of the article
}

C Biomed Central (c) 2014 Thomas et al.; licensee BioMed Central Ltd. This is an Open Access article distributed under the terms of the Creative Commons Attribution License (http://creativecommons.org/licenses/by/4.0), which permits unrestricted use, distribution, and reproduction in any medium, provided the original work is properly credited. The Creative Commons Public Domain Dedication waiver (http://creativecommons.org/publicdomain/zero/1.0/) applies to the data made available in this article, unless otherwise stated. 


\section{Background}

\section{Cognitive behavior therapy (CBT) for psychosis}

The continued prevalence of medication-resistant psychotic symptoms has prompted an interest in developing psychological treatments as an adjunct to medication to improve symptoms. Cognitive behavior therapy (CBT) has been the dominant therapy approach applied to psychosis, aiming to reduce the distress and disability associated with psychotic symptoms such as hallucinations and delusions. Whilst CBT for Psychosis (CBTp) includes a number of facets [1,2], the core intervention characterising this approach is belief modification (cognitive restructuring) - the ' $\mathrm{C}$ ' in $\mathrm{CBT}$ [3]. This involves modifying the content of anomalous beliefs identified as being associated with distress and dysfunction such as delusions and other symptom-related cognitions that may lead to distress, e.g. the belief that hallucinated voices have the power to harm the patient. The major techniques for weakening such beliefs include challenging the evidence for them, generating alternative explanations, identifying irrational or inconsistent elements and constructing reality testing experiments $[4,5]$.

The efficacy of CBTp for symptom improvement in schizophrenia initially showed considerable promise. Several early meta-analyses reported large and important clinical effects [6-8] providing key evidence that CBTp was an effective treatment for positive psychotic symptoms. This led to CBTp becoming an established evidence-based treatment for residual psychotic symptoms [1,9] and it has been recommended for routine provision in clinical practice guidelines now for many years [10-12]. With the advent of more rigorous trials and further meta-analyses however, the initial optimism about the impact of CBT has become increasingly cautious [3,13]. Recent reviews have concluded that CBT has only a small effect on symptoms $[14,15]$ and questioned its advantages over other less complex therapies [16,17], although this has also been vigorously debated (e.g., $[18,19])$.

Even so, CBTp is limited by being a challenging therapy to deliver. Around half the patients who receive CBTp in clinical trials fail to attain clinically significant benefits in symptoms [5], suggesting this approach is only suitable for some patients. Failure to respond has been associated with pre-therapy measures of resistance to considering alternatives to delusions [20], denying any possibility of being mistaken [21], and the patient failing to engage with the therapist's model of reality during the therapy process [22]. These observations suggest that the partial effectiveness of existing psychological treatment arose from some patients not being amenable to the process of belief modification which is so central to CBT. Even for those patients who are amenable to cognitive restructuring, modifying psychotic beliefs is usually a slow and difficult endeavour [23]. In order to avoid the risk of psychological reactance - a defensive restitution or even strengthening of the belief [24,25] - cognitive restructuring must proceed slowly and gently, and only once several sessions have been spent building up a strong therapeutic relationship $[4,5,23]$. A minimum duration of therapy of six months is recommended [10], with briefer interventions producing changes in mood symptoms but failing to impact upon psychotic symptomatology $[10,26]$. In addition, because this process is highly complex, it requires a high level of skill in CBT. Accordingly, some trials have observed poorer outcomes with less experienced therapists $[27,28]$.

Thus, in spite of the initial apparent success of CBTp, there remains much room to improve the psychological management of psychotic symptoms. In particular, approaches that are less reliant upon belief modification to bring about therapeutic change may prove to be briefer, more effective, cost-effective, and more readily disseminated into routine practice.

\section{Acceptance and commitment therapy (ACT) for psychosis}

Acceptance and commitment therapy (ACT) is a manualised psychological treatment $[29,30]$ with a clear theoretical basis [31] that shows promise as an alternative approach to psychological intervention in psychosis. According to the ACT model, psychological problems develop through the inappropriate or unhelpful regulation of behavior through language processes leading to psychological inflexibility in relation to environmental contingencies [32]. Rather than effecting change through modification of belief content, ACT aims to reduce the extent to which beliefs and other symptoms dominate conscious experience and behavior. More specifically, treatment of symptoms is not focused on their removal, but on taking them less literally and disrupting their link with behavior. ACT uses experiential exercises, illustrative metaphors and behavioral tasks in order to effect change, with logical analysis having a relatively minor role [33]. Although ACT is often described as a variant of CBT, the overlap in therapeutic elements is small [1].

As the name suggests, ACT has two broad components. In the Acceptance component, methods cultivating cognitive defusion, contact with the present moment and self as the observer of experience help the individual recognise and dispassionately observe symptoms and associated reactions, rather than believing and acting on them. The Commitment component emphasises the articulation of personal values and goals, and seeks to minimise the effects of symptoms on achieving those goals in behavioral terms. At its core, ACT is a behavioral treatment $[29,30]$, grounded in producing functional change.

Two limited RCTs have tested the application of ACT to acute psychosis with surprisingly powerful outcomes. Bach and Hayes [34] assessed the impact of a brief 
version of ACT on symptoms in a population of 80 inpatients with positive psychotic symptoms. Rate of rehospitalisation was used as a concrete and objective measure of the negative functional impact of symptoms. In contrast to CBTp, which involves an average of 20 sessions [35], treatment involved just four 45-minute sessions. The authors found that, compared with a treatment-as-usual control group, participants in the ACT group had half the rate of rehospitalisation ( $20 \%$ vs $40 \%$ ) over a follow-up period of four months.

Gaudiano and Herbert [36] conducted a second trial of ACT for psychosis, providing approximately three hours of ACT to inpatients with schizophrenia and implementing an enhanced treatment-as-usual comparison condition. This trial additionally examined outcomes using validated symptom measures including the Brief Psychiatric Rating Scales (BPRS). Although there were no overall group differences on the BPRS from pre-test to follow-up, half of the participants randomised to receive ACT improved two standard deviations or more on the BPRS, compared with only about $10 \%$ of participants receiving usual ward-based therapy. In terms of functional change, participants in the ACT group reported significantly less social disruption from their symptoms compared to controls. Rehospitalisation rates showed a similar trend to the Bach and Hayes trial (28\% vs 45\%), although this difference failed to reach significance in this smaller sample $(n=40)$. However, a recent analysis of pooled data from both trials supported the original reduced rate of rehospitalisation, which was mediated by reduced symptom believability [37].

Our group earlier conducted an RCT $(n=43)$ of a 15session psychological intervention for medication-resistant command hallucinations called "Treatment of Resistant Command Hallucinations (TORCH)", which incorporates elements of ACT alongside CBTp [38]. While the results showed no strong evidence that the combined ACT-CBT treatment targeting command hallucinations was superior to the Befriending control condition, the $n$ of 43 in this trial was considerably below what would now be considered advisable based on effect sizes published after the trial's completion [39]. When we examined the pattern of within group findings and conducted comparisons with a waitlist control, results suggested that both interventions had benefit with a different pattern of outcomes observed across the two conditions. While Befriending was primarily effective in the short-term for distress [38], TORCH showed both short and longer-term benefit for a broader range of outcomes including illness severity.

The TORCH study was rigorously conducted including blinding and an active control. Notwithstanding its low power, outcomes were in line with the more recent evaluations of CBTp indicating no substantial benefit for symptoms compared to other psychological treatments including those which may be less sophisticated such as Befriending. However, given this trial involved a combined CBT-ACT treatment targeted to command hallucinations it is not possible to draw conclusions about the efficacy of ACT alone and also its efficacy in relation to psychotic symptoms in general. In contrast, while the initial trials of pure ACT for psychosis were positive they were not blinded and they did not have an active comparison condition. Thus, there is a need to conduct a rigorous trial of pure ACT for psychosis to test whether the promise shown in initial trials holds under more stringent conditions.

\section{Aim, hypothesised outcomes and mechanisms of change}

The aim of this study was to test the efficacy of ACT for psychosis in a sample of community-residing patients with chronic medication-resistant psychotic symptoms by comparison with an active comparison condition. The study of ACT in this population is important because they represent the majority of service users living with psychosis. In doing so, we hypothesised a number of effects of ACT on specific symptom-related outcomes and processes that would be consistent with the ACT model.

As with CBT, ACT does not aim to eliminate psychotic symptoms, but to reduce resultant distress and disability to enable better quality of life. Both hallucinations and delusions appear to be multidimensional phenomena, in which different dimensions may show significant variation between individuals and over time, e.g., frequency, degree of preoccupation and degree of conviction in content, as well as resultant distress and impact upon behavior $[40,41]$. The dimensions of preoccupation and conviction appear particularly important in determining levels of disability and subjective distress. Indeed, in the general population, beliefs with the same themes as delusions may be held in attenuated form without interfering with social functioning or being found significantly distressing $[42,43]$. The dimensions of preoccupation and conviction appear more important than content itself in distinguishing patients with schizophrenia from non-patients who hold unconventional beliefs $[42,43]$. The cognitive defusion techniques used in the Acceptance component of ACT provide an avenue for reducing the level of preoccupation and conviction with psychotic symptoms, but, in contrast to $\mathrm{CBT}$, without requiring modification of the content of beliefs themselves. The ACT model proposes that preoccupation with distressing internal experiences such as psychotic symptoms is maintained by the paradoxical effect of repeatedly engaging in unsuccessful attempts to avoid and suppress them $[29,30]$. The Acceptance component of ACT involves exercises to help the person recognise the futility of trying to directly suppress and control such experiences, combined with teaching skills in mindful observation of experiences in a detached manner without attempting to change or judge 
them. The use of defusion techniques is associated with increased tolerance of experimentally induced pain, as opposed to distraction or no instruction [32], suggesting that this is applicable to aversive internal experiences in general. In addition, there is evidence that defusion techniques are particularly effective in reducing repetitive cognitive processes such as depressive rumination, anxious worry and persistence of obsessive thoughts (e.g., $[32,44])$. These findings suggest that such techniques may also be helpful for reducing preoccupation with distressing delusions [45]. Further, there is a strong argument that distancing and acceptance techniques may also be specifically applicable to preoccupation with auditory hallucinations given their 'verbal' form, their compelling salience, and the propensity of voice hearers to be drawn into reacting to the literal content $[46,47]$. In support of this contention, several strands of research have found acceptance strategies to be helpful in managing voices (see [39] for review).

Defusion techniques are also hypothesised to have an impact on conviction related to both delusional beliefs and hallucinatory content. Bach and Hayes [34] found reduced ratings of the 'believability' of delusions and voice content post-treatment relative to controls, and Gaudiano and Herbert [48] found that changes in rated believability of voice content predicted reduction in distress, independent of changes in frequency of hallucination.

Although ACT may lead to reductions in preoccupation and conviction with psychotic symptoms, thereby impacting on resultant distress and disruption to life, it is unlikely to have an effect on how often symptoms are experienced. In the Bach and Hayes [34] and Gaudiano and Herbert [36] trials, no differences were observed in reported frequency of symptoms post-treatment between participants receiving ACT and treatment-as-usual. However, a noteworthy paradoxical effect was observed in the Bach and Hayes trial: relative to controls, participants in the ACT group were less likely to report that symptoms had completely remitted. Furthermore, those ACT participants who reported still experiencing symptoms had better outcomes in that they were significantly less likely to be readmitted. This paradoxical association of fewer reports of symptom remission with otherwise positive outcomes compared with controls may be explained by control participants being more likely to deny symptoms with the acceptance component of ACT being protective against this [34]. Denial of the psychotic experience is associated with a "sealing over" recovery style [49]. Those who seal over tend to isolate the psychotic experience and are disinterested in any exploration of symptoms [49]. In contrast, those with an "integrative" recovery style are curious to understand and explore their illness. This study will therefore examine whether ACT results in reduced sealing over of psychotic experiences and determine whether this accounts for any paradoxical increase in symptom reporting. It is hypothesised that if any paradoxical increase in the number of symptoms reported occurs, this will be mediated by recovery style.

The Commitment component of ACT involves exercises to help the patient clarify their personal values and encouraging commitment to take action in line with these values. It is expected that this will lead to adaptive behavioral change, reducing the impact of negative symptoms and improve social functioning. It might be noted that White et al. [50] found a reduction in negative symptoms in a feasibility trial of ACT for emotional dysfunction following psychosis. However, the very small sample size and apparent baseline differences makes it difficult to draw strong conclusions from this finding.

Figure 1 summarises the expected pathways of action for ACT. Acceptance and defusion processes are expected to lead to reduced preoccupation, conviction, distress and disruption to life associated with hallucinations and/or delusions resulting in a general improvement in positive symptoms. Increased commitment to valued action is expected to lead to adaptive behavioral change and a general improvement in negative symptoms. The net outcome of these changes will be observed in improvements in overall mental state and social functioning.

Our hypotheses are therefore that, compared to patients receiving equivalent clinician time, patients who receive ACT will show improvement in (a) overall mental state (incorporating positive, negative and general symptoms); (b) preoccupation, conviction, distress and disruption to life associated with positive psychotic symptoms; (c) social functioning, and (d) adaptive behavior. Outcomes will also be examined in relation to (e) service utilisation. We hypothesise that all changes will be achieved by the conclusion of therapy and maintained at six-month follow-up. The study will also examine hypothesised mechanisms of action, namely that within the ACT group (f) improvements in positive symptoms will be predicted by reductions in experiential avoidance, (g) improvements in negative symptoms will be predicted by increased commitment to action and (h) if any paradoxical increase in the number of symptoms are reported, this will be mediated by recovery style. Finally, we considered that cognitive impairments commonly seen in schizophrenia [51-53] could interfere in understanding abstract metaphors and retaining information in the ACT condition, thereby moderating outcomes. The inclusion of several cognitive measures as covariates, including verbal memory, abstract reasoning and premorbid IQ, will allow us to examine the role of cognitive functioning on outcome.

\section{Methods/Design \\ Trial design}

The study is a prospective, single (rater)-blind, RCT using parallel group comparison design involving the 


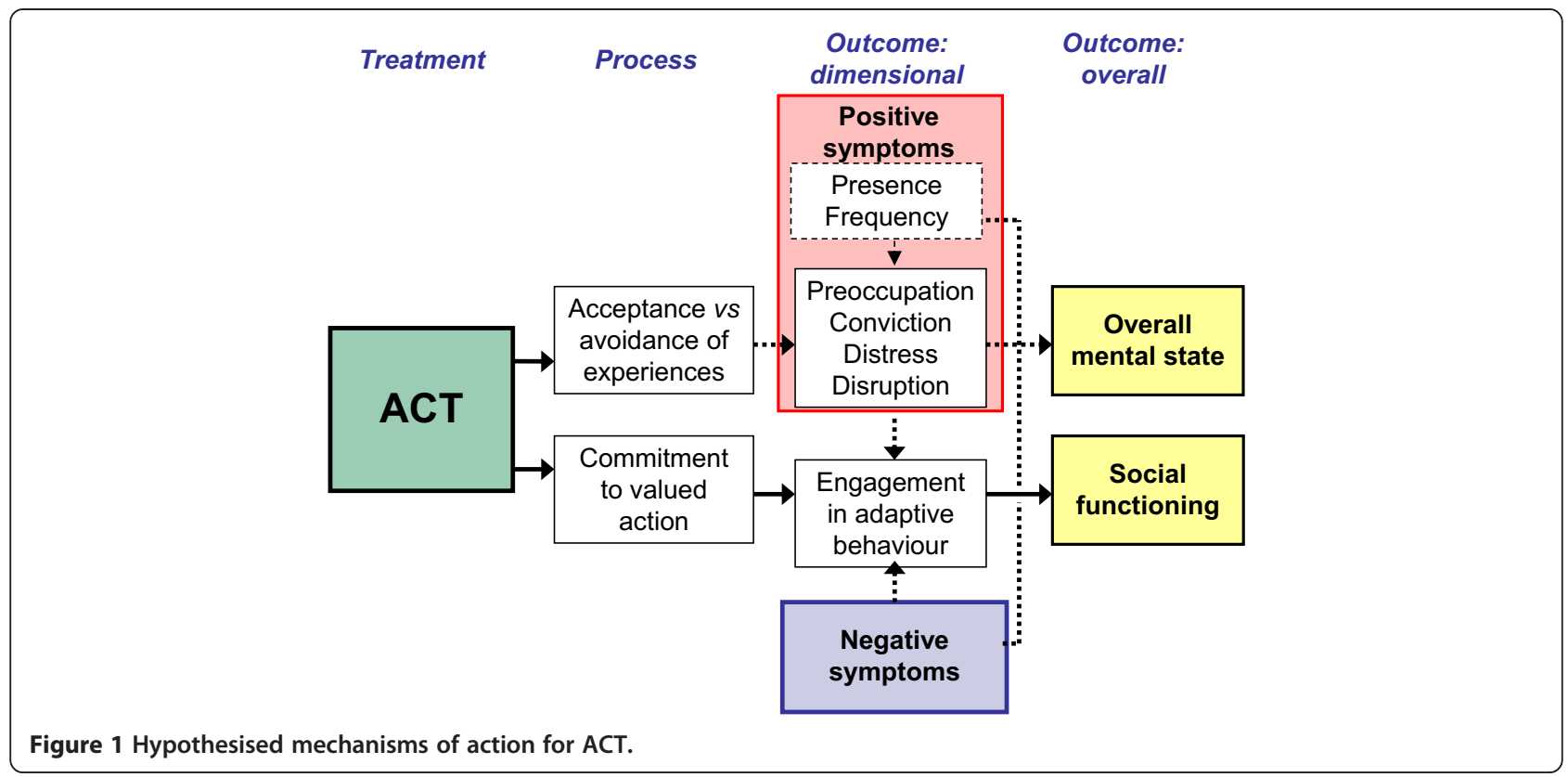

intervention, $\mathrm{ACT}$, and a comparison condition, Befriending. Both types of intervention are provided by all therapists involved in the trial. Participants continue their usual treatment during the course of their participation in the trial. Assessments take place prior to commencing therapy (baseline), at the completion of therapy (endpoint), and at 6 months follow-up. The study is being conducted in compliance with the Helsinki Declaration and has been approved by the following governing ethics committees: Alfred Hospital Ethics Committee (273/09); Austin Health Human Research Ethics Committee (H2009/03570); Eastern Health (E13/1011); La Trobe University Human Ethics Committee (07-160); Melbourne Health Mental Health Research and Ethics Committee (2007.39); Mercy Health \& Aged Care Human Research Ethics Committee R08/34 W); Peninsula Health Human Research \& Ethics Committee (HREC/11/PH/4); Southern Health (now Monash Health) Human Research Ethics Committee A (08084A); St. Vincent's Hospital Human Research Ethics Committee (045/08); and The Melbourne Clinic Research Ethics Committee (185). Written informed consent is required from all participants.

\section{Sample}

\section{Selection criteria}

Inclusion criteria include: (a) age between 18 and 65 years inclusive; (b) a current diagnosis of schizophrenia or schizoaffective disorder, according to the Structured Clinical Interview for DSM-IV-TR Axis I Disorders [54]; (c) current residual hallucinations or delusions which are associated with significant distress or disability (a score of 4 or greater on items P1 and/or P3 of the Positive and Negative Syndrome Scale (PANSS - [55]); (d) these symptoms have been present continuously over the past six months; and (e) have been on antipsychotic medication at doses within the therapeutic range over the past six months. Exclusion criteria are: (f) any neurological disorder that may affect cognitive function; (g) insufficient conversational English for meaningful participation; (h) having an IQ less than 70 , as estimated by the Wechsler Test of Adult Reading (WRAT - [56]); (i) any change in antipsychotic medication within the previous eight weeks or planned at the time of intake; (j) currently receiving other formal psychological treatment.

\section{Sample size}

We determined that a sample of 53 participants per treatment arm would enable endpoint between group effects for overall mental state of $d=0.55$ or greater to be detected with $80 \%$ power $(\alpha=0.05)$. The effect size chosen is a little lower than the $d=0.60$ reported in the TORCH [38] and Gaudiano and Herbert [36] trials for overall mental state.

\section{Recruitment and randomisation}

Participants are recruited from public mental health services and private providers including non-government psychiatric disability rehabilitation services in metropolitan Melbourne, Australia. Potential participants are identified via clinician referral, supplemented by review of clinician case lists and advertising within services and print media to generate self-referrals. Participants who provide written informed consent undertake an interview to complete the baseline assessment measures prior to randomisation and confirm eligibility. 
The randomisation materials and procedure was prepared at study inception by an independent statistician who subsequently had no further involvement in the project. Randomisation is stratified by site of recruitment (9 sites) and recovery style (integration or sealing over). Within each of the 18 factorial combinations of site of recruitment and recovery style, a random permuted blocks procedure was used to generate a list of As and Bs. The procedure was implemented in Microsoft Excel, and a high quality random number generator was used to choose each random sequence of blocks, without replacement within each sequence. For each of the 18 groups a numbered pile of sealed opaque envelopes was prepared, each envelope containing a slip of paper marked 'A (ACT)' or 'B' (Befriending), in accord with the generated sequence. The printing on the slips is very faint, and slips were folded so that no hint of the A or B can be seen through the envelope even when held up to light. Using the envelopes in numbered order, for the appropriate group, gives an allocation of subjects to treatment A or B in accordance with CONSORT guidelines. The random number seed, and the 18 printed sequences of As and Bs, are sealed in double envelopes, with again, no hint of the contents being visible through the envelopes. Assignment of participants to conditions using these materials is undertaken by a research assistant who works independently of staff involved in the recruitment, assessment and management of participants in the study. Group allocation is revealed to the trial therapists and by letter to the participant and their primary treating clinician.

\section{Treatments}

$A C T$

Participants randomised to the ACT condition are offered eight 50-minute sessions of ACT, delivered at weekly to fortnightly intervals within a time frame of around three months. We considered that an eight-session intervention would provide a more comprehensive treatment for our chronically affected sample than that offered in the two trials with acute inpatients, but would nevertheless remain much briefer than trials of CBTp, which average 20 sessions in length [35], and our combined ACT-CBT TORCH protocol of 15 sessions [38]. ACT is conducted according to a local manual which is largely based on the first edition of the ACT manual [29] but with recommended adaptations for psychosis [34,57]. The 8 sessions comprise an initial assessment session followed by 7 intervention sessions. The initial session includes an orientation to therapy and an assessment of symptoms and problems related to psychosis. During this session, the therapist also notes the participant's degree of struggle with symptoms and motivation for change as basic indicators of the most useful starting point for subsequent interventions. For example, creative hopelessness, the traditional beginning to $\mathrm{ACT}$, is likely to be most suitable for clients who have struggled with their symptoms and are highly motivated to change. For clients who are less motivated or ambivalent about therapy it may be more useful to start with a focus on values and goals. While guidelines are provided, the therapist is expected to tailor the components according to the needs of the participant as identified in the assessment phase. The order in which these intervention components are delivered is also left to the therapist's judgment. Participants are provided with folders for handouts and sessions are recorded onto CDs for home review.

\section{Befriending}

Participants randomised to the control condition are offered eight 50-minute sessions of the Befriending intervention [58], a fully manualised treatment previously used as a control condition in trials of psychological intervention in schizophrenia (e.g., [59]), including our TORCH trial [38]. Befriending involves engaging in conversation about everyday topics, whilst explicitly avoiding discussion of symptoms, problems or emotive issues. It has been shown to provide the same amount of therapist engagement and expectancy as CBT and to have similar drop-out rates [60].

In both conditions, therapy is provided by clinical psychologists with experience of psychological interventions in schizophrenia and with additional training in $\mathrm{ACT}$ and Befriending. They attend weekly peer supervision led by JF with SH providing advice and assistance in dealing with complex ACT-related issues through monthly supervision via videoconferencing. Medication, case management and other aspects of treatment continue to be managed by local services. At the end of treatment, a summary report is provided to the primary treating clinician.

\section{Measures}

\section{Outcome measures}

Overall mental state Overall impact upon mental state is measured by the Positive and Negative Syndrome Scale (PANSS - [55]), a widely-used, comprehensive interviewbased measure of schizophrenia symptomatology which consists of seven items measuring positive symptoms, seven items measuring negative symptoms and 16 items measuring general symptoms. We will report overall mental state (as measured by the total score) along with subscale scores.

Positive symptoms The Psychotic Symptom Rating Scales (PSYRATS - [41]) is a semi-structured interview designed to assess the severity of an individual's auditory hallucinations and delusions across a range of physical and psychological dimensions. We use items from the PSYRATS to assess the severity of hallucinations and 
delusions according to the specific dimensions of interest to the study which include preoccupation, conviction, distress and disruption to life. In order to be able to control for the presence and frequency of symptoms, the frequency dimension of the PSYRATS auditory hallucinations subscale and the amount of preoccupation dimension of the PSYRATS delusions subscale is also be assessed. This scale has good psychometric properties and excellent inter-rater reliability across both scales is excellent, ranging from 0.788-1.00 for hallucinations and $0.884-1.00$ for delusions [41].

Delusions are further assessed using the Peters Delusions Inventory (PDI - [43]). This self-report measure assesses delusional thinking more broadly and has been found to be a reliable and valid of delusional thinking in the general population and to discriminate between clinical and non-clinical samples on conviction, preoccupation and distress [43]. Participants are asked to indicate the presence or absence of 21 delusional beliefs (e.g., "Do you ever feel as if people are reading your mind?") giving a total score of $0-21$. Each delusional belief endorsed is then rated on dimensions of preoccupation, conviction and distress. The total number of items endorsed on the PDI will be used to assess for any paradoxical increases in symptom reporting.

Social functioning Impact on overall social functioning is assessed with the Social Functioning Scale (SFS - [61]). This is a comprehensive interview-based assessment for individuals with schizophrenia that assesses seven areas of functioning essential to living in the community (social engagement, interpersonal behavior, prosocial activities, recreation, independence and employment). It has been shown to be valid, reliable, and sensitive to change [61]. This is completed on the basis of interview with the participant and discussion with their case manager or alternative informant. The Sheehan Disability Scale (SDS - [62]) is a 3-item self-report instrument measuring impairment and functional disability due to psychiatric symptoms across key domains of work/education, social life and family life/home responsibilities. Responses are measured on a 10-point partially anchored visual analogue scale from 0 (not at all) to 10 (extremely) and assessed over the last month.

Adaptive behavior Direct impact on behavior is assessed using a time budget measure for schizophrenia [63] which calculates the level of adaptive activity undertaken by the participant over a representative week on the basis of a detailed interview. Level of adaptive activity is rated from 0 : nothing - lying thinking sleeping sitting etc. to 4: time period filled with a variety of demanding independent activities requiring significant motivation and planning and with some variation in tasks, e.g., work. Four time blocks per day are rated over 7 days with the total weekly score ranging from $0-112$. It has been used successfully with schizophrenia, with good reliability and validity [63]. The specific hypothesis that treatment will moderate the impact of negative symptoms will be assessed using the PANSS Negative Syndrome subscale.

Service utilisation Service utilisation during the followup period will be compared with baseline rates of service utilisation using a subset of self-report questions extracted from instrumentation as used in the previous (2007) Australian National Survey of Mental Health and Wellbeing. Forms of service use include both psychiatric hospital admissions (number and days) and consultations with mental health service providers (number and length). This broader measure of service use is used in preference to rehospitalisation rates alone, due to the relatively low baseline rate of readmission in a chronic sample. Indeed, Gumley et al. [64] estimate a sample size of over 400 participants would be required to detect changes in readmission rates, which was not feasible in this study.

\section{Process measures}

Acceptance and commitment The 16-item Acceptance and Action Questionnaire (AAQ - [65]) is used in order to measure the hypothesised therapy mechanisms of (a) increasing the degree of acceptance of psychotic experiences as opposed to attempted suppression and experiential avoidance, and (b) increasing commitment to valued action. Like the more commonly used 9-item AAQ, this version has single factor structure. It was chosen on the basis of the authors' view ([58], p. 563) that in a research context the additional items were likely to confer greater sensitivity to change. (The present study was devised prior to publication of the AAQ II [66]). Internal consistency of the 16-item version was not reported however it correlates .89 with the 9 -item version which has a Cronbach alpha of .70. Extensive convergent and divergent validity has been reported with versions of the measure widely used in research on the mechanisms of ACT [32,65]. As this measure has not been previously applied to psychosis, our group has previously developed a measure for auditory and command hallucinations that directly taps each construct (the Voices Acceptance and Action Scale - VAAS), with promising initial results and acceptable psychometric properties [67]. The VAAS is used in addition to the AAQ for participants experiencing auditory hallucinations.

Recovery style The Recovery Style Questionnaire (RSQ [68]) is used to assess the degree to which participants 'integrate' their illness, acknowledging their illness experiences with interest and curiosity (e.g., "I can see positive aspects to my illness"; "My illness is part of me"), as 
opposed to 'seal over' whereby participants seek to encapsulate their psychosis as separate from themselves and express disinclination for any exploration of their illness experiences (e.g., "My illness has had little effect on my life"; "I'm not really interested in my illness"). This self-report scale has 39 items; higher scores indicate greater sealing over. The RSQ has been evaluated as a valid measure of recovery style according to the original concept $[49,69]$. It has been found to have high test-retest reliability (Spearman $4=0.81$ ) and an acceptable Cronbach's alpha of 0.73 [68].

\section{Covariates}

Premorbid IQ The WTAR is administered as a test of premorbid IQ. Participants are asked to read a list of 50 words that have atypical grapheme to phoneme translations (e.g., "liaison", " paradigm") and are scored according to accuracy of pronunciation (raw score range 0-50). WTAR raw scores are standardised based on age then converted to the predicted WAIS-III IQ. While predicted IQ scores will be reported descriptively, these are associated with very large confidence intervals; standardised scores will therefore be used for analyses due to their greater precision.

Abstract verbal reasoning The Similarities subscale of the Wechsler Adult Intelligence Scale III (WAIS-III) [70] is administered to assess abstract verbal reasoning in relation to concept formation. The subscale consists of 19 items: participants are asked to describe how two given things are alike (e.g., "table and chair", "poem and statue"). Raw scores are converted to age-adjusted scaled scores using the normative data provided in the manual.

Verbal memory The Story Memory and Story Recall subtests of the Repeatable Battery for the Assessment of Neuropsychological Status (RBANS) [71] are used to assess immediate and delayed memory. Story Memory involves reading a brief story and asking the participant to repeat it back immediately. It is scored over two trials (total score 0-24). Story Recall involves free recall of the story after a delay of 15-20 minutes (total score 0-12). Raw scores will be converted to $\mathrm{z}$ scores for analyses using normative data for each participant's age group [72].

Antipsychotic medication Antipsychotic medication changes and dosages are tracked at each assessment point and will be converted to chlorpromazine-equivalent dosages [73]. Chlorpromazine-equivalent dosages will be compared between groups in order to verify whether groups are equivalent, and correlations will be examined between dosage changes during the study period and clinical outcomes in order to exclude the possibility that medication changes are responsible for outcome.

\section{Therapy evaluation}

At the end of their post-therapy assessment, participants are asked to complete the Client Satisfaction Questionnaire-8 (CSQ-8 - [74]) to assess the acceptability and experience of therapy. As well, four additional questions give participants an opportunity to provide more specific feedback about their experience of therapy. These include ratings of emotional response and problem improvement with respect to problems related to psychosis and qualitative feedback related to therapy gains and disappointments. We expect that therapy will be rated positively by a majority of patients.

\section{Treatment fidelity}

Treatment sessions are audio recorded and a stratified random sample of audio files will be rated by an independent assessor for compliance with therapy condition. The assessor, blinded to treatment condition, will be required to assign each audio file to either ACT or Befriending. Because no validated scale currently exists to measure therapist adherence to ACT guidelines more specifically, an ACT fidelity scale is developed in the initial phase of the trial. The Befriending Treatment Integrity Measure [58] is used to assess the quality of Befriending sessions and to ensure that ACT sessions do not include Befriending techniques.

\section{Primary outcome}

The primary outcome is overall mental state as measured using the PANSS total score. PANSS subscale scores for positive, negative and general scales will also be reported.

\section{Secondary outcomes}

Secondary outcomes are: the PSYRATS subscales (preoccupation, conviction, distress, and disruption to life) adjusting for the presence and frequency of symptoms; the SFS; and service utilisation.

\section{Procedure}

Assessments are conducted by research assistants trained in administration of the measures at baseline (prior to randomisation), at the end of therapy approximately three months following baseline (endpoint), and at six months following the end of therapy (follow-up). Outcome measures, process measures and medication are assessed at all three time points except for service utilisation which is administered only at baseline and follow-up. Cognitive measures are done where possible at Time 1 or, if necessary, at a later time point.

\section{Blindness}

Considerable efforts are made to ensure that the blindness of raters is maintained. Offices, data storage and travel logs of raters and therapists are kept separate. Participants 
and clinical staff are regularly reminded not to divulge details of their therapy to the raters and a reminder sign placed prominently in front of the participant during assessments. Blindness is assessed after endpoint and follow-up assessments by asking raters classify participants into treatment condition and to indicate their level of confidence. Breaches in blindness are recorded and addressed by changing the rater wherever possible.

\section{Statistical analysis}

The primary focus of the analysis is differential changes in the ACT group versus Befriending from baseline to endpoint, which will be analysed within the framework of Treatment Group (ACT vs. Befriending) by Measurement Occasion (baseline, endpoint, follow-up) design. Analyses will be undertaken using mixed-model repeated measures (MMRM) which is the recommended method for examining clinical trial data [75]. Primary evidence of the efficacy of ACT will be a significant two-way interaction demonstrating greater change in outcome measures in the ACT group from pre- to post-therapy. Baseline to followup interactions with treatment group will also be examined. Where there are significant group $\mathrm{x}$ time interactions, planned contrasts will compare changes from baseline under each intervention at endpoint and follow-up.

Intention-to-treat (ITT) analysis [76] will be used. MMRM is well-suited to ITT analyses because this approach uses all available information from subjects as randomised to produce ITT estimates of treatment effects under only mild assumptions concerning the nature of withdrawal [75]. Most importantly, subjects with incomplete data are not discarded and missing data are not replaced with unprincipled estimated values or observations carried forward [77]. This approach is regarded as the most appropriate method of analysing RCT data by the highest quality journals, which have adopted the CONSORT standard (see, e.g., [78]). However, if data are grossly non-normal and cannot be successfully transformed, non-parametric methods will be used.

The mediational impact of pre to post AAQ, RSQ and medication dose change will be assessed using the Sobel test [79], arguably the most powerful current method of detecting indirect effects [80]. This test assesses the statistical significance of the product of the coefficients for treatment-mediator and mediator-outcome effects. A bootstrapped multivariate extension of the Sobel test described by Preacher and Hayes $[81,82]$ will be used, which is suitable for non-parametric data, and allows us to examine both the total indirect effect and the individual effect of each specified mediator, controlling for the other.

\section{Discussion}

This protocol describes the first RCT of ACT in chronic medication-resistant psychosis. It is also the first RCT of
$\mathrm{ACT}$ in psychosis to use an active comparison group, and be designed to fully meet CONSORT criteria. It thus rises to the challenge of critics both of ACT trials, such as Öst [83], who point out the scarcity of well-controlled trials of the therapy across many client groups, and critics of CBTp trials who note variable trial quality $[13,15]$ and the negative correlation between trial quality and strength of outcome $[13,84]$. If successful, it will be the most definitive trial to date of ACT for people living with psychosis.

ACT for psychosis holds promise as a therapy that may compare favourably with CBTp in outcomes, uptake by patients, treatment duration and ease and costs of training. By adopting an alternative therapeutic method, ACT may show greater applicability to psychosis than CBT, or may prove to be more suitable for some patients who currently fail to respond to CBT. Additionally, from its explicit emphasis on reducing behavior in response to symptoms and promotion of behavior in line with values, ACT may be more successful than CBT in reducing negative symptoms and symptom-related disability [50]. In addition, its grounding of treatment in the individual's values is likely to provide an approach which is highly acceptable to patients and consistent with the recovery framework promoted by consumers and adopted by mental health services in many countries. Furthermore, by following a set manualised structure, rather than relying upon the complex and lengthy process of belief modification, therapy can be much briefer ( 8 sessions ACT vs. an average of 20 sessions CBTp) and potentially practiced by a wider range of clinicians. ACT provides an approach which is more cost-effective and more readily manualised than existing psychological treatments: this may lead to it being more widely disseminated into routine practice, and ultimately lead to greater availability of effective treatment to consumers.

\section{Abbreviations}

AAQ: Acceptance and action questionnaire; ACT: Acceptance and commitment therapy; BPRS: Brief psychiatric rating scales; CBT: Cognitive behavior therapy; CBTp: Cognitive behavior therapy for psychosis; CSQ-8: Client satisfaction questionnaire-8; DSM-IV_TR: Diagnostic and statistical manual of mental disorders $4^{\text {th }}$ edition, text revision;

IQ: Intelligence quotient; ITT: Intention to treat; MMRM: Mixed-model repeated measures; PANSS: Positive and negative syndrome scale; PDI: Peter's delusions inventory; PSYRATS: Psychotic symptom rating scales; RBANS: Repeatable battery for the assessment of neurological status; RCT: Randomised controlled trial; RSQ: Recovery style questionnaire; SDS: Sheehan disability scale; SFS: Social functioning scale; TORCH: Treatment of resistant command hallucinations; VAAS: Voices acceptance and action scale; WAIS: Wechsler adult intelligence scale; WTAR: Wechsler test of adult reading.

Competing interests

The authors declare that they have no competing interests.

\section{Authors' contributions}

All authors participated in the design of the trial. All authors read and approved the final manuscript. 


\section{Acknowledgements}

This trial is funded by the National Health and Medical Council of Australia (Grant 487335).

The trial is undertaken through La Trobe University.

\section{Author details}

${ }^{1}$ School of Health Sciences, Swinburne University, Hawthorn, Victoria 3122 Australia. ${ }^{2}$ Monash Alfred Psychiatry Research Centre, Melbourne, Victoria 3004, Australia. ${ }^{3}$ Department of Psychiatry, Monash University, Clayton, Victoria 3800, Australia. ${ }^{4}$ School of Psychological Science, La Trobe University, Melbourne, Victoria 3086, Australia. ${ }^{5}$ Department of Psychiatry, University of Melbourne, Parkville, Victoria 3052, Australia. ${ }^{6}$ St Vincent's Hospital Mental Health, Fitzroy, Victoria 3065, Australia. ${ }^{7}$ Office of the Vice-Chancellor and Discipline of Psychiatry, Monash University, Clayton, Victoria 3800, Australia. ${ }^{8}$ Department of Psychiatry, University of Melbourne, Parkville, Victoria 3010, Australia. ${ }^{9}$ Florey Institute of Neuroscience and Mental Health, Parkville, Victoria 3052, Australia. ${ }^{10}$ Department of Psychology, University of Nevada, Reno, Nevada 89557, USA. ${ }^{11}$ NorthWestern Mental Health, Royal Melbourne Hospital, Melbourne, Victoria 3050, Australia.

Received: 4 June 2014 Accepted: 7 July 2014

Published: 11 July 2014

\section{References}

1. Dickerson FB, Lehman AF: Evidence-based psychotherapy for schizophrenia. J Nerv Ment Dis 2006, 194(1):3-9.

2. Morrison AP, Barratt S: What are the components of CBT for psychosis? A Delphi study. Schizophr Bull 2010, 36(1):136-142.

3. Gaudiano BA: Cognitive Behavior Therapies for Psychotic Disorders: Current Empirical Status and Future Directions. Clin Psychol Sci Pr 2005, 12(1):33-50.

4. Chadwick P, Birchwood M, Trower P: Cognitive Therapy for Delusions, Voices and Paranoia. Chichester: John Wiley \& Sons; 1996.

5. Garety P, Fowler D, Kuipers E: Cognitive-Behavioral Therapy for medication-resistant symptoms. Schizophr Bull 2000, 26(1):73-86.

6. Gould RA, Mueser KT, Bolton E, Mays V, Goff D: Cognitive therapy for psychosis in schizophrenia: an effect size analysis. Schizophr Res 2001, 48:335-342.

7. Pilling S, Bebbington P, Kuipers E, Garety P, Geddes J, Orbach G, Morgan C: Psychological treatments in schizophrenia: I. Meta-analysis of family intervention and cognitive behaviour therapy. Psychol Med 2002, 32:763-782.

8. Rector N, Beck AT: Cognitive behavioral therapy for schizophrenia: an empirical review. J Nerv Ment Dis 2001, 189:278-287.

9. Jones C, Cormac I, Silveira-da-Mota-Neto Il, Campbell C: Cognitive behaviour therapy for schizophrenia. Cochrane Database Syst Rev 2004 4:CD000524. http://onlinelibrary.wiley.com/doi/10.1002/14651858.CD000524. pub2/pdf.

10. National Institute for Clinical Excellence: Schizophrenia. London: NICE; 2002

11. National Institute for Clinical Excellence: Psychosis and Schizophrenia in Adults: Treatment and Management. London: NICE; 2014.

12. Royal Australian and New Zealand College of Psychiatrists clinical practice guidelines team for the treatment of schizophrenia and related disorders: Royal Australian and New Zealand College of Psychiatrists clinical practice guidelines for the treatment of schizophrenia and related disorders. Aust NZ J Psychiat 2005, 39(1-2):1-30.

13. Tarrier N, Wykes $\mathrm{T}$ : Is there evidence that cognitive behaviour therapy is an effective treatment for schizophrenia? A cautious or cautionary tale? Behav Res Ther 2004, 42:1377-1401.

14. Jauhar S, McKenna PJ, Radua J, Fung E, Salvador R, Laws KR: Cognitivebehavioural therapy for the symptoms of schizophrenia: systematic review and meta-analysis with examination of potential bias. Brit J Psychiat 2014, 204(1):20-29.

15. Lynch D, Laws KR, McKenna PJ: Cognitive behavioural therapy for major psychiatric disorder: does it really work? A meta-analytical review of well-controlled studies. Psychol Med 2010, 40:9-24.

16. Jones C, Hacker D, Cormac I, Meaden A, Irving CB: Cognitive behaviour therapy versus other psychosocial treatments for schizophrenia. Cochrane Database Syst Rev 2012, 4:CD008712. http://onlinelibrary.wiley. com/doi/10.1002/14651858.CD008712.pub2/pdf.
17. Jones C, Hacker D, Cormac I, Meaden A, Irving CB: Cognitive Behavior Therapy Versus Other Psychosocial Treatments for Schizophrenia. Schizophr Bull 2012, 38(5):908-910.

18. Birchwood MJ, Shiers D, Smith J: CBT for psychosis: not a quasi-neuroleptic. Brit J Psychiat 2014, http://bjp.rcpsych.org/content/204/1/20/reply\#bjprcpsych_ el_55218.

19. Byrne RE: RE: Cognitive-behavioural therapy for the symptoms of schizophrenia. Brit J Psychiat 2014, http://bjp.rcpsych.org/content/204/1/20/ reply\#bjprcpsych_el_55218.

20. Chadwick P, Lowe CF: Measurement and modification of delusional beliefs. J Consult Clin Psych 1990, 58(2):225-232.

21. Garety P, Fowler D, Kuipers E, Freeman D, Dunn G, Bebbington P, Hadley C, Jones S: London-East Anglia randomized controlled trial of cognitivebehavioural therapy for psychosis. II: Predictors of outcome. Brit $J$ Psychiat 1997, 171:420-426.

22. McGowan JF, Lavender T, Garety P: Factors in outcome of cognitivebehavioural therapy for psychosis: Users' and clinicians' views. Psychol Psychother Theor Res Pract 2005, 78:513-529.

23. Cather C, Penn D, Otto M, Goff D: Cognitive therapy for delusions in schizophrenia: models, benefits, and new approaches. J Cognit Psychother 2004, 18(3):207-221.

24. Brehm JW: A Theory of Psychological Reactance. New York: Academic Press; 1966.

25. Milton F, Patwa VK, Hafner RJ: Confrontation vs belief modification in persistently deluded patients. Brit J Med Psychol 1978, 51:127-130.

26. Turkington D, Kingdon D, Turner T: Effectiveness of a brief cognitivebehavioural therapy intervention in the treatment of schizophrenia. Brit J Psychiat 2002, 180:523-527.

27. Wykes T, Hayward P, Thomas N, Green N, Surguladze S, Fannon D, Landau S: What are the effects of group cognitive behaviour therapy for voices? A randomised control trial. Schizophr Res 2005, 77(2-3):201-210.

28. Durham RC, Guthrie M, Morton RV, Reid D, Treliving LR, Fowler D, MacDonald RR: Tayside-Fife clinical trial of cognitive-behavioural therapy for medication-resistant psychotic symptoms. Brit J Psychiat 2003, 182:303-311.

29. Hayes SC, Strosahl KD, Wilson KG: Acceptance and Commitment Therapy: An Experiential Approach to Behavior Change. New York: The Guilford Press; 1999

30. Hayes SC, Strosahl KD, Wilson KG: Acceptance and Commitment Therapy: An Experiential Approach to Behavior Change. 2nd edition. New York: The Guilford Press; 2013.

31. Barnes-Holmes $Y$, Hayes SC, Barnes-Holmes D, Roche B: Relational frame theory: A post-Skinnerian account of human language and cognition. In Advances in Child Development and Behavior. Edited by Reese HW, Robert K. San Diego, CA: Academic Press; 2001:101-138.

32. Hayes SC, Luoma JB, Bond FW, Masuda A, Lillis J: Acceptance and commitment therapy: model, processes and outcomes. Behav Res The 2006, 44(1):1-25

33. Hayes SC: Acceptance and Commitment Therapy, Relational Frame Theory, and the third wave of behavior therapy. Behav Ther 2004, 35(4):639-665.

34. Bach P, Hayes SC: The use of Acceptance and Commitment Therapy to prevent rehospitalization of psychotic patients: a randomized controlled trial. J Consult Clin Psych 2002, 70(5):1129-1139.

35. Turkington D, Dudley R, Warman DM, Beck AT: Cognitive-behavioural therapy for schizophrenia: a review. J Psychiat Prac 2004, 10(1):5-16.

36. Gaudiano BA, Herbert JD: Acute treatment of inpatients with psychotic symptoms using Acceptance and Commitment Therapy: pilot results. Behav Res Ther 2006, 44:415-437.

37. Bach P, Gaudiano BA, Hayes SC, Herbert JD: Acceptance and commitment therapy for psychosis: intent to treat, hospitalization outcome and mediation by believability. Psychosis 2013, 5(2):166-174.

38. Shawyer F, Farhall J, Mackinnon A, Trauer T, Sims E, Ratcliff R, Larner C, Thomas N, Castle D, Mullen P, Copolov D: A randomised controlled trial of acceptance-based cognitive behavioural therapy for command hallucinations in psychotic disorders. Behav Res Ther 2012, 50:110-121.

39. Shawyer F, Farhall J: Acceptance-based CBT for command hallucinations: rationale, implementation, outcomes and reflections from the TORCH project. In Acceptance and Mindfulness in the Treatment of Psychosis: Current Trends and Future Directions. Edited by Gaudiano BA. New York: Oxford University Press; in press 
40. Brett-Jones J, Garety PA, Hemsley D: Measuring delusional experiences: a method and its application. Brit J Clin Psychol 1987, 26:257-265.

41. Haddock G, McCarron J, Tarrier N, Faragher EB: Scales to measure dimensions of hallucinations and delusions: the psychotic rating scale (PSYRATS). Psychol Med 1999, 29:879-889.

42. Peters E, Day S, McKenna J, Orbach G: Delusional ideation in religious and psychotic populations. Br J Clin Psychol 1999, 38(Pt 1):83-96.

43. Peters $E_{1}$ Joseph S, Day S, Garety P: Measuring delusional ideation: the 21-item Peters et al. Delusions Inventory (PDI). Schizophr Bull 2004, 30(4):1005-1022

44. Singer AR, Dobson KS: An experimental investigation of the cognitive vulnerability to depression. Behav Res Ther 2007, 45(3):563-575.

45. Thomas N: A model for the development of acceptance and mindfulness based therapies: Preoccupation with psychotic experiences as a treatment target. In Acceptance and Mindfulness in the Treatment of Psychosis: Current Trends and Future Directions. Edited by Gaudiano BA. New York: Oxford University Press; in press.

46. Farhall J, Greenwood KM, Jackson HJ: Coping with hallucinated voices in schizophrenia: a review of self-initiated strategies and therapeutic interventions. Clin Psychol Rev 2007, 27(4):476-493.

47. Shawyer F, Farhall J, Sims EF, Copolov D: Command hallucinations in psychosis: acceptance and disengagement as a focus of treatment. In Theory and Practice in Contemporary Australian Cognitive and Behaviour Therapy: Proceedings of the 28th National AACBT Conference. Edited by Jackson M, Murphy G. Melbourne: Australian Association for Cognitive Behaviour Therapy; 2005:5-14

48. Gaudiano BA, Herbert JD: Believability of Hallucinations as a Potential Mediator of Their Frequency and Associated Distress in Psychotic Inpatients. Behav Cogn Psychoth 2006, 34(04):497-502.

49. McGlashan TH, Levy ST, Carpenter WT: Integration and sealing over: clinically distinct recovery styles from schizophrenia. Arch Gen Psychiat 1975, 32:1269-1272.

50. White R, Gumley A, McTaggart J, Rattrie L, McConville D, Cleare S, Mitchell G: A feasibility study of Acceptance and Commitment Therapy for emotional dysfunction following psychosis. Behav Res Ther 2011, 49(12):901-907.

51. Caspi A, Reichenberg A, Weiser M, Rabinowitz J, Kaplan Z, Knobler H, Davidson-Sagi N, Davidson M: Cognitive performance in schizophrenia patients assessed before and following the first psychotic episode. Schizophr Res 2003, 65(2-3):87-94.

52. Fioravanti $M$, Bianchi $V$, Cinti M: Cognitive deficits in schizophrenia: an updated metanalysis of the scientific evidence. BMC Psychiatry 2012, 12(1):64

53. Tandon R, Keshavan M, Nasrallah H: Schizophrenia, "Just the Facts": What we know in 2008 Part 1: Overview. Schizophr Res 2008, 100(1-3):4-19.

54. First MB, Spitzer RL, Gibbon M, Williams JBW: Structured Clinical Interview for DSM-IV-TR Axis I Disorders-Patient Edition (SCID-I/P, 1/2007 revision). New York, New York: Biometrics Research Department, New York State Psychiatric Institute; 2007.

55. Kay SR: Positive and Negative Syndromes in Schizophrenia. New York: Brunner/Mazel; 1991.

56. Wechsler D: Wechsler Test of Adult Reading. New York: Psychological Corporation; 2001

57. Bach PA: ACT with the seriously mentally ill. In A Practical Guide to Acceptance and Commitment Therapy. Edited by Hayes SC, Strosahl KD. New York: Springer; 2005.

58. Bendall S, Killackey E, Jackson HJ, Gleeson J: Befriending Manual. Melbourne: ORYGEN Research Centre, University of Melbourne; 2003.

59. Sensky T, Turkington D, Kingdon D, Scott JL, Scott J, Siddle R, O'Carroll M, Barnes TRE: A randomized controlled trial of cognitive-behavioural therapy for persistent symptoms in schizophrenia resistant to medication. Arch Gen Psychiat 2000, 57(2):165-172.

60. Bendall S, Jackson HJ, Killackey E, Allot K, Johnson T, Harrigan S, Gleeson J, McGorry PD: The credibility and acceptability of Befriending as a control therapy in a randomized controlled trial of Cognitive Behaviour Therapy for acute first episode psychosis. Behav Cog Psychoth 2006 34:277-291

61. Birchwood M, Smith J, Cochrane R, Wetton S, Copestake S: The Social Functioning Scale. The development and validation of a new scale of social adjustment for use in family intervention programmes with schizophrenic patients. Br J Psychiat 1990, 157:853-859.

62. Sheehan DV, Harnett-Sheehan K, Raj BA: The measurement of disability Int Clin Psychopharm 1996, 11(Suppl 3):89-95.
63. Jolley S, Garety PA, Ellett L, Kuipers E, Freeman D, Bebbington PE, Fowler DG, Dunn G: A validation of a new measure of activity in psychosis. Schizophr Res 2006, 85(1-3):288-295.

64. Gumley A, O'Grady M, McNay L, Reilly J, Power K, Norrie J: Early intervention for relapse in schizophrenia: results of a 12-month randomized controlled trial of cognitive behavioural therapy. Psychol Med 2003, 33(3):419-431.

65. Hayes SC, Strosahl KD, Wilson KG, Bissett RT, Pistorello J, Toarmino D, Polusny M,A, Dykstra TA, Batten SV, Bergan J, Stewart SH, Zvolensky MJ, Eifert GH, Bond FW, Forsyth JP, Karekla M, McCurry SM: Measuring experiential avoidance: a preliminary test of a working model. Psychol Rec 2004, 54(4):553-578.

66. Bond FW, Hayes SC, Baer RA, Carpenter KM, Guenole N, Orcutt HK, Waltz T, Zettle RD: Preliminary psychometric properties of the Acceptance and Action Questionnaire-II: a revised measure of psychological inflexibility and experiential avoidance. Behav Ther 2011, 42(4):676-688.

67. Shawyer F, Ratcliff R, Mackinnon A, Farhall J, Hayes SC, Copolov D: The Voices Acceptance and Action Scale (VAAS): pilot data. J Clin Psychol 2007, 63(6):593-606.

68. Drayton M, Birchwood M, Trower P: Early attachment experience and recovery from psychosis. Br J Clin Psychol 1998, 37(Pt 3):269-284.

69. McGlashan $\mathrm{TH}$ : Recovery style from mental illness and long-term outcome. J Nerv Ment Dis 1987, 175(11):681-685.

70. Wechsler D: Wechsler Adult Intelligence Scale-Third Edition (WAIS-III). San Antonio: The Psychological Corporation; 1997.

71. Randolf C: Repeatable Battery for the Assessment of Neuropsychological Status. San Antonio: The Psychological Corporation; 1998.

72. Randolf C: Repeatable Battery for the Assessment of Neuropsychological Status (RBANS). Supplement 1. Harcourt Assessment; 2006. http://images. pearsonclinical.com/images/PDF/technical_reports/RBANS.pdf.

73. Gardner DM, Murphy AL, O'Donnell H, Centorrino F, Baldessarini RJ: International consensus study of antipsychotic dosing. Am J Psychiat 2010, 167(6):686-693.

74. Attkisson CC, Zwick R: The Client Satisfaction Questionnaire: psychometric properties and correlations with service utilization and psychotherapy outcome. Eval Program Plann 1982, 5(3):233-237.

75. Gueorguieva R, Krystal JH: Move over ANOVA: progress in analyzing repeated-measures data and its reflection in papers published in the Archives of General Psychiatry. Arch Gen Psychiat 2004, 61(3):310-317.

76. Sheiner LB, Rubin DB: Intention-to-treat analysis and the goals of clinical trials. Clin Pharmacol Ther 1995, 57(1):6-15.

77. Houck PR, Mazumdar S, Koru-Sengul T, Tang G, Mulsant BH, Pollock BG, Reynolds CF 3rd: Estimating treatment effects from longitudinal clinical trial data with missing values: comparative analyses using different methods. Psychiat Res 2004, 129(2):209-215.

78. Bruce ML, Ten Have TR, Reynolds CF 3rd, Katz II, Schulberg HC, Mulsant BH, Brown GK, McAvay GJ, Pearson $U$, Alexopoulos GS: Reducing suicidal ideation and depressive symptoms in depressed older primary care patients: a randomized controlled trial. JAMA 2004, 291(9):1081-1091.

79. Sobel ME: Asymptotic confidence intervals for indirect effects in structural equations models. Sociol Methodol 1982, 13:290-312

80. MacKinnon DP, Lockwood CM, Hoffman JM, West SG, Sheets V: A comparison of methods to test mediation and other intervening variable effects. Psychol Methods 2002, 7(1):83-104.

81. Preacher KJ, Hayes AF: SPSS and SAS procedures for estimating indirect effects in simple mediation models. Behav Res Methods Instrum Comput 2004, 36(4):717-731.

82. Preacher KJ, Hayes AF: Asymptotic and resampling strategies for assessing and comparing indirect effects in multiple mediator models. Behav Res Methods 2008, 40(3):879-891.

83. Ost LG: Efficacy of the third wave of behavioral therapies: a systematic review and meta-analysis. Behav Res Ther 2008, 46:296-321.

84. Wykes T, Steel C, Everitt B, Tarrier N: Cognitive Behavior Therapy for schizophrenia: effect sizes, clinical models, and methodological rigor. Schizophr Bull 2008, 34(3):523-537.

doi:10.1186/1471-244X-14-198

Cite this article as: Thomas et al:: A randomised controlled trial of acceptance and commitment therapy (ACT) for psychosis: study protocol. BMC Psychiatry 2014 14:198. 